\title{
La Villa des Alleux à Taden. Lectures archéologique et architecturale d'un établissement rural de la cité des Coriosolites
}

Patrick Galliou

\section{(2) OpenEdition \\ 1 Journals}

\section{Édition électronique}

URL : https://journals.openedition.org/abpo/4673

DOI : $10.4000 / a b p o .4673$

ISBN : 978-2-7535-7968-2

ISSN : $2108-6443$

\section{Éditeur}

Presses universitaires de Rennes

\section{Édition imprimée}

Date de publication : 20 novembre 2019

Pagination : 201-202

ISBN : 978-2-7535-7966-8

ISSN : 0399-0826

\section{Référence électronique}

Patrick Galliou, « La Villa des Alleux à Taden. Lectures archéologique et architecturale d'un établissement rural de la cité des Coriosolites ", Annales de Bretagne et des Pays de l'Ouest [En ligne], 126-3 | 2019, mis en ligne le 20 novembre 2019, consulté le 08 janvier 2022. URL : http://journals.openedition.org/abpo/ 4673 ; DOI : https://doi.org/10.4000/abpo.4673 


\section{Comptes rendus}

FERRETTE, Romuald (dir.), La Villa des Alleux à Taden. Lectures archéologique et architecturale d'un établissement rural de la cité des Coriosolites, Rennes, PUR, coll "Archéologie et Culture ", 2018, 205 p., illustrations couleur et noir et blanc.

Souvent déclenchées par des travaux d'aménagement ou de remembrement qui menaçaient les vestiges antiques, les fouilles de villae, avec publication partielle ou extensive de leurs résultats, furent relativement nombreuses dans l'Ouest de la Bretagne au cours des années 1960-1970. Par la suite, l'archéologie historique bretonne, pour des raisons que l'on ne songera pas à discuter, se détourna, pour une large part, de l'exploration de ces établissements ruraux pour se consacrer à l'étude de quartiers d'agglomérations romaines (Rennes, Vannes, Quimper, etc.), en avant d'une expansion urbaine généralisée. En raison des progrès apportés aux techniques de fouille et d'identification des mobiliers au cours des cinquante dernières années, il a paru nécessaire d'ouvrir à nouveau le dossier des villae romaines de la péninsule armoricaine. On ne peut donc que se réjouir de la publication, sous la direction de Romuald Ferrette, de la fouille de la villa des Alleux en Taden (Côtes-d'Armor). D'autres verront probablement le jour dans les années à venir.

Le premier chapitre de l'ouvrage retrace les étapes de la découverte du site, décrit le cadre topographique et géologique où se situait la villa ainsi que le déroulement de l'intervention archéologique, lors de l'hiver 2005-2006, et analyse brièvement le phasage de l'occupation du site, qui fut habité pendant trois siècles environ. Est enfin passé en revue le corpus des villae fouillées en Bretagne depuis le XIX ${ }^{\mathrm{e}}$ siècle.

Le chapitre suivant analyse les premières occupations du site (phase I). Les plus anciens vestiges sont ceux de trois salles, au plan incomplet, bâties sur sablières basses et poteaux porteurs, que les maigres fragments de céramique associés permettent de dater d'une période s'étendant des années 40 apr. J.-C. au dernier tiers du même siècle. Lors de la phase suivante (phase II), difficilement datable mais appartenant peut-être à la fin de ce siècle, les premiers bâtiments, vraisemblablement construits en bois et en terre, furent rasés, et l'on dressa sur le même emplacement un édifice rectangulaire, avec soubassements en pierres sèches, d'environ $16 \mathrm{~m}$ de long sur 13,30 m de large. Cette petite structure bâtie comporte sept pièces et l'on peut être tenté d'y voir un bâtiment à galerie de façade (pièce E6), encadrée par deux " celliers " (pièces E5 et E7). La phase qui suit (phase III - état I), du II siècle, vit un considérable accroissement de l'occupation, la construction des premiers bains marquant la recherche d'un certain confort. L'ajout de nombreuses salles - environ une vingtaine - au bâtiment précédent, constituant le cœur du nouvel édifice, dessine une structure en $\mathrm{U}$, deux ailes de longueur inégale enserrant une cour centrale. Les premiers bains étaient placés dans l'aile sud. Quelques aménagements ponctuels, apportés peu de temps après, ne modifièrent pas la physionomie globale de l'ensemble. 
La phase IV, appartenant également au II ${ }^{\mathrm{e}}$ siècle, constitue incontestablement un tournant dans l'histoire de la villa et l'apogée de son développement, un nouveau programme architectural accroissant la surface bâtie par des reprises des ailes sud et nord et ouest et la mise en place d'un portique à trois branches autour de la cour orientale, sans doute occupée en partie par un jardin et fermée par un mur périphérique, la cour occidentale, vraisemblablement à usage agricole, étant probablement bordée par des bâtiments de service. C'est également au cours de cette phase que fut installé, dans le balnéaire de l'aile sud, un décor peint à fresque. Une analyse architecturale complète des structures exhumées clôt ce chapitre.

Les phases suivantes (V à vIII) voient des modifications sensibles être apportées à la villa de phase Iv. Lors de la phase v (vers 150-200 apr. J.-C.), l'aile thermale fut restructurée et sa fonction première abandonnée, tandis que des transformations diverses étaient opérées dans le corps occidental de l'ensemble. Au cours de la phase suivante (avant 250 apr. J.-C.), l'établissement paraît avoir été partiellement abandonné ou seulement occupé de manière sporadique, tandis que des matériaux étaient prélevés ici et là dans les structures bâties, parfois même partiellement démolies (aile sud). Les phases VII et vIII, postérieures à l'époque romaine, correspondent enfin à l'abandon définitif de la villa et à l'implantation d'enclos dont l'orientation diffère radicalement de celle des bâtiments antiques.

Comme le soulignent les auteurs, la fouille des Alleux montre l'évolution, assez classique dans l'Ouest de la Gaule, d'un bâtiment rectangulaire simple (phases I et II) à une villa à galerie de façade à plan en U, précédée à l'est d'une cour d'agrément et doublée à l'ouest d'une pars rustica à usage agricole (phases III), puis à une villa "à plan compact " (phase Iv). Une discussion de cette évolution, appuyée sur des exemples régionaux et extra-régionaux, ainsi qu'une analyse serrée de la probable utilisation des pièces et des espaces de circulation apportent un intéressant éclairage sur l'histoire de la villa et la manière de vivre de ses habitants, les comparaisons de l'ensemble de la phase Iv aux villae du même type de l'Ouest de la Gaule, à vrai dire assez peu nombreuses, permettant de juger de la place de l'établissement des Alleux dans le tissu social et économique des Coriosolites. Le déclin de la villa, ou plutôt le changement de ses paramètres fonctionnels, dans la phase $\mathrm{v}$, est très justement rapporté à un phénomène très répandu dans les campagnes - mais aussi les villes - de Gaule du Nord, où, dans la seconde moitié du III ${ }^{\mathrm{e}}$ siècle, l'on voit progressivement disparaître les symboles de la romanité, sans que ce reflux soit dû à une véritable crise économique et encore moins à des incursions barbares. Il semble néanmoins plus précoce ici qu'ailleurs, sans que l'on puisse en donner l'explication. La pars rustica ayant été largement détruite par la construction d'une route, il est difficile de préciser les fonctions agricoles de l'établissement des Alleux, mais l'on peut raisonnablement supposer qu'elles reposaient, comme ailleurs, sur l'élevage et la culture des céréales. Il est enfin manifeste, comme le soulignent les auteurs, que cette villa était un établissement somme toute modeste, dont les relations avec un environnement qui n'est guère plus riche restent encore à explorer. En dépit des incertitudes qui demeurent encore quant à la villa des Alleux, due pour une part à la destruction d'une partie de ses bâtiments et de l'autre à l'étonnante maigreur du mobilier archéologique (une seule monnaie!), ce travail de qualité apporte de nombreux et utiles renseignements sur un établissement rural coriosolite, tout en relançant le nécessaire débat quant à la place des villae dans le paysage rural de l'Armorique romaine et à leur évolution au fil des siècles. On en recommandera donc la lecture à tous ceux qu'intéresse cette période de notre passé. 